KfK 3859

Dezember 1984

\title{
Nonsteady End Effects in Hele-Shaw Cells
}

J. N. Koster, P. Ehrhard, U. Müller Institut für Reaktorbauelemente

\section{Kernforschungszentrum Karlsruhe}



KERNFORSCHUNGSZENTRUM KARLSRUHE

INSTITUT FÜR REAKTORBAUELEMENTE

KfK 3859

Nonsteady end effects in Hele-Shaw ce11s

J.N. Koster*, P. Ehrhard, U. Müller

* present address: NASA Lewis, MS 501-8, Cleveland, OH 44135 and University of Colorado at Boulder, USA 
Als Manuskript vervielfältigł

Für diesen Bericht behalten wir uns alle Rechte vor

Kernforschungszentrum Karlsruhe $\mathrm{GmbH}$

ISSN 0303-4003 


\section{Abstract}

The evolution of convective flow in a finite Hele-Shaw cell is presented. Two new results were found. First, at the same Rayleigh number there coexist three different flow states in separated regions of the fluid layer, the center region having a steady flow and the two end regions exhibiting timedependent flow. Second, the flow oscillations in the two end regions are monoperiodic and are never correlated. There is no transition to a more complicated oscillation observed. The time-dependent flow is bound at low and high Rayleigh number by transitions to steady state flow.

\section{Instationäre Randeffekte in einer Hele-Shaw Zelle.}

\section{Zusammenfassung}

Es werden Konvektionsexperimente in einer endichen Hele-Shaw Zelle vorgestellt. Zwei wesentliche neue Ergebnisse wurden hierbei gefunden. Zum ersten zeigte sich, daB bei gleicher Rayleighzahl drei ijrtliche Bereiche existieren, wobei in der Mitte der Zelle stationäre und an zwei Rändern zeitabhängige Strömungsformen existieren. Zum zweiten konnte gezeigt werden, dab die Oszillationen an beiden Rändern monoperiodisch und nicht korreliert (d.h. unabhängig voneinander) sind. Ein Ubergang zu komplexeren Strömungsformen konnte nicht beobachtet werden. Vielmehr waren die oszillatorischen Strömungsformen, sowohl zu niederen als auch zu höheren Rayleighzahlen hin, durch stationäre Formen eingegrenzt. 


\section{Contents}

$\underline{\text { Page }}$

1. Introduction

1

2. Experimental set up and procedures

3. Experimental results

6

3.1 Onset of convection

6

3.2 Steady convection

6

3.3 Oscillatory convection

3.4 Reverse transition

4. Discussion

5. Summary

6. References 


\section{Introduction}

Time dependent convective flow is a timely subject to study the behaviour of differential equations and the fluid dynamics of turbulence. Our goal will be to understand the second topic: the physics of the development of oscillatory convection.

It is known today that in large containers, i.e. systems of many degrees of freedom, the oscillations are nonperiodic at the onset of time dependent flow. An evaluation of this work has been given by Busse (1981) and Koschmieder $(1981)$.

Ahlers \& Behringer (1978), Dubois \& Berge (1980), Gollub \& Benson (1980), Libchaber \& Maurer (1980) and others found out that in small boxes, i.e. systems of few degrees of freedom, the oscillations are more ordered. They observed that mostly the oscillations begin as a monoperiodic oscillation. At higher temperature differences transitions occur to a biperiodic, nonperiodic or other type of oscillations exhibiting several subharmonics and/or intermittencies.

In large Hele-Shaw cells, Koster \& Müller (1982) obtained results similar to those found in large boxes where the oscillations develop as more or less noisy ones. They visualized also a pronounced interaction of the convection rolls. In smaller Hele-Shaw slots, Koster \& Müller (1984) found a series of transitions between different types of oscillation.

Busse \& Whitehead (1974) and Berge \& Dubois (1976) have shown that in high Prandt 1 number fluids unstable thermal boundary layers drive the time dependent flow. Krishnamurt1 (1970) and Koster \& Müller (1982) reconfirmed this result for Hele-Shaw cells. Howard's (1964) idea of an unstable thermal boundary layer causing the oscillations can thus be accepted as the essential starting mechanism of the time dependent convection for high Prandt number fluids.

However, it is less well understood why a time dependent convection flow exhibits under certain conditions periodic, quasiperiodic, frequency locked or nonperiodic oscillations and moreover undergoes transitions between these types of oscillations. The major question is what causes the transitions and 
can separate flow osctllators be identified. It appears that flow visualization is necessary to investigate this problem. Dubois \& Berge (1980) reported interferometric results showing that one oscillator develops in a plume and a second one in the thermal boundary layer. It was shown also by Dubois \& Berge (1981) that each cell in a two cell configuration may exhibit one oscillator. Koster \& Muller (1984) could not confirm the first observation but found some cvidence for the second one.

The present experiment will be directed towards finding uncorrelated oscillators which would support the idea (Koster \& Muiler, 1984) that individual frequencies of unstable thermal boundary layers drive the oscillations and cause the transitions between different oscillatory states.

\section{Experimental set up and procedures}

Apart from problem ortented modifications, the Hele-Shaw cell is similar to those described by Koster \& Müller (1982, 1984). The vertical walls of the cell were made of $4 \mathrm{~mm}$ thick Plexiglas which categorizes the cell as a low conductivity cell (see Koster \& Muller, 1984). To improve the linearity of a vertical kemperature profile in the sidewalls, the Plexiglas cell was sandwiched by two BK7-glass windows in thermal contact to the upper and lower isothermal boundaries. The dimensions of the Hele-Shaw cell are, gap width $\mathrm{d}=4.1 \mathrm{~mm}$, height $\mathrm{h}=40.3 \mathrm{~mm}$, length $\mathrm{b}=111.2 \mathrm{~mm}$. The aspect ratios are $h / b=0.36$ and $h / d=9.83$ or $h / b / d=0.36 / 1 / 0.037$. The optical pathway through the Plexiglas cell is $Y=2.95$, which indicates that the amount of Plexiglas fringes that appear in the interferograms are not hindering the interpretation. The fluid was silicone oil of viscosity $\nu=20 \mathrm{cSt}$ at $\mathrm{T}=20{ }^{\circ} \mathrm{C}$ and Prandt 1 number $\mathrm{Pr} \simeq 235$.

The Hele-Shaw cell is built into a low conductivity frame (Novotex) which has windows for optical access. Further, the framed cell is insulated with $30 \mathrm{~cm}$ thick partly removable heat insulation material to reduce the heat transfer with the surroundings.

The fluid layer was heated from below and cooled from above with a symmetric heating rate of $0.02 \mathrm{k} / \mathrm{min}$. The heating periods lasted from about 10 to 30 minutes. Between these periods, equilibration periods of between 30 minutes 
and 24 hours were provided. The vertical thermal diffusion times of silicone oil and Plexiglas were taken into account in this way.

The temperature difference $\Delta \mathrm{T}$ and the upper temperature $\mathrm{T}_{1}$ were measured in order to calculate the Rayleigh number which is defined as

$$
R \mathbf{a}=g B \Delta h^{3} /(\nu K)
$$

with acceleration of gravity $g$, thermal expansion coefficient $\beta$, vertical temperature difference $\Delta \mathrm{T}$ across the layer of height $\mathrm{h}$, the kinematic viscosity $v$ and the thermal diffusivity $k$. The Rayleigh number is normalized as $\mathrm{Ra}^{*}=\mathrm{Ra} / \mathrm{Ra}$ osc, where $\mathrm{Ra}$ osc is the critical Rayleigh number of the onset of oscillatory convective flow. The Prandtl number is defined as

$$
\operatorname{Pr}=\nu / K \text {. }
$$

The locations of the thermocouples are shown to scale in figure 1. At the upper and at the lower side there are 4 thermocouples each side of $0.25 \mathrm{~mm}$ diameter which protrude about $2 \mathrm{~mm}$ into the fluid. Four of these thermocouples measure temperature differences in the fluid and four others measure local temperatures referenced to the icepoint. These thermocouples should provide information on temperature fluctuations in the thermal boundary layers of individual convective roll cells. 


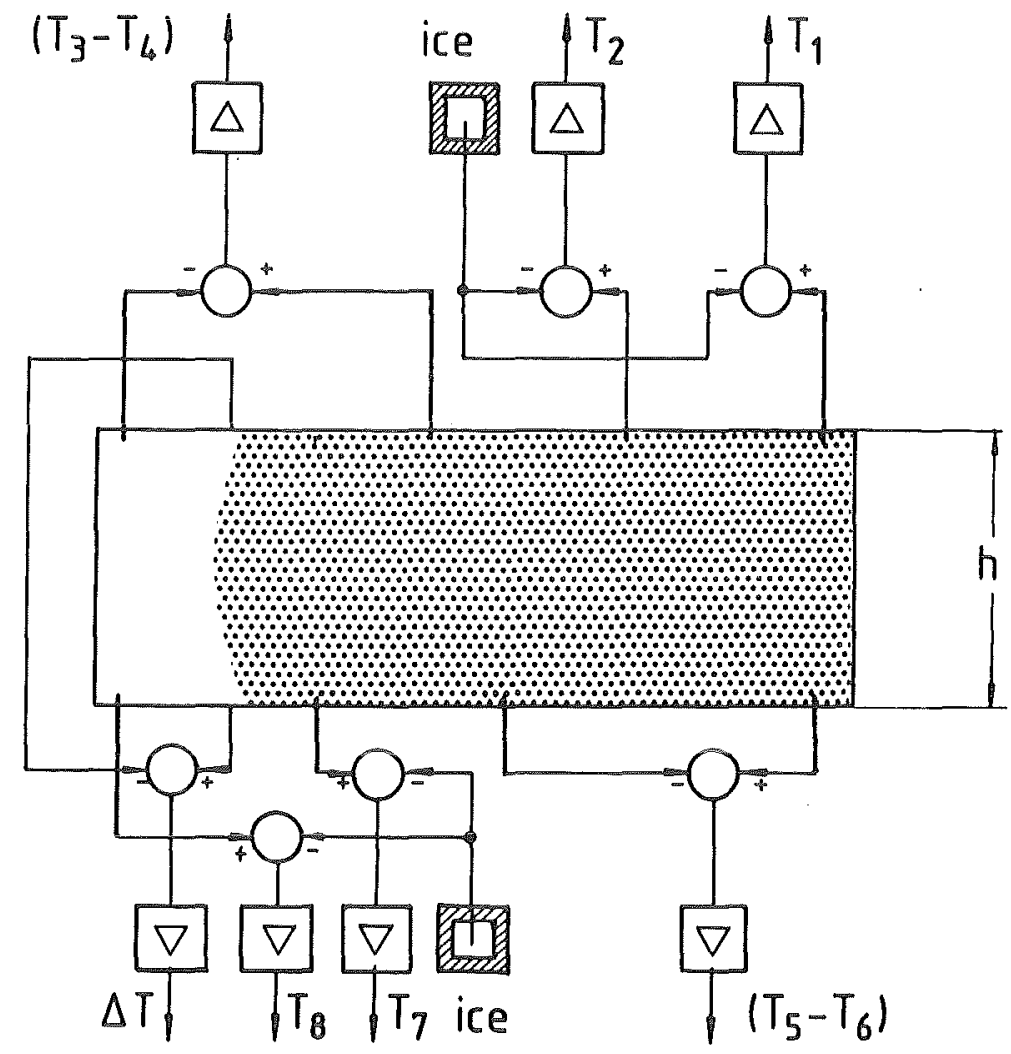

Hele-Shaw Cell h/b/d $=0.36 / 1 / 0.037$

Fig. 1: Sketch of the area hxb of the Hele-Shaw cell with thermocouple locations. Shaded area represents the visualized area. 
Data registration for time series analysis of the oscillatory flow were made at constant temperature difference between the horizontal boundaries. The total of six time dependent signals from the horizontal thermal boundary layers were amplified, filtered at a frequency of $10 \mathrm{~Hz}$ and their dc of was removed before storing on tape. Segments of 2048 values were digitized with a time step of $2 \mathrm{~s}<\Delta t<5 \mathrm{~s}$. Then the auto- respectively cross-correlation coefficient as well as the auto- and cross-power density spectra of different signal combinations were calculated. The spectra provide single frequencies, of which the basic ones are evaluated as dimensionless periods

$$
\tau=\frac{k}{f \cdot h^{2}}
$$

which is a measure of the ratio of the period of oscillation and the thermal diffusion time of the liquid.

As a criterion of transition we consider a thermal boundary layer as stable, when the thermocouple in that layer does not register time dependent temperatures. We consider it unstable if there is a time dependent signal.

For a detalled error analysis of this investigation we refer to that of Koster \& Müller (1984) which we consider valid for this experiment. 


\section{Experimental results}

The following results originate from two experiments in one Hele-Shaw cell.

\subsection{Onset of convection}

The threshold of the onset of convection was measured in several runs and defined as $R_{C}=3.9 \times 10^{5}(\Delta T=1.35 \mathrm{~K})$. This value fits into the theoretical frame given by Frick \& Clever (1980). In one experiment a four roll flow pattern developed and in the second one there existed a five roll pattern. It was observed that the wavenumber of the end rolls is higher than the wavenumber of the center rolls. The following ratios of the critical wavenumbers were measured:

$$
\begin{aligned}
& \text { 5-rol1-experiment } \\
& \qquad \alpha_{e} / \alpha_{c}=5.83 / 5.73=0.67 \\
& \text { 4-rol1-experiment } \\
& \alpha_{e} / \alpha_{c}=5.27=0.75
\end{aligned}
$$

Here the indices $e$ and $c$ have the meaning of $e=$ end cell and $c=$ center cell.

\subsection{Steady convection}

After the onset of convection the Rayleigh number is increased by symmetric heating and cooling the lower and upper boundaries in a quasisteady way. Typical interferograms, showing qualitative temperature fields with increasing temperature difference are shown in figure 2 and described below.

From these fringe patterns qualitative streamlines are evaluated and shown along with the interferograms. 

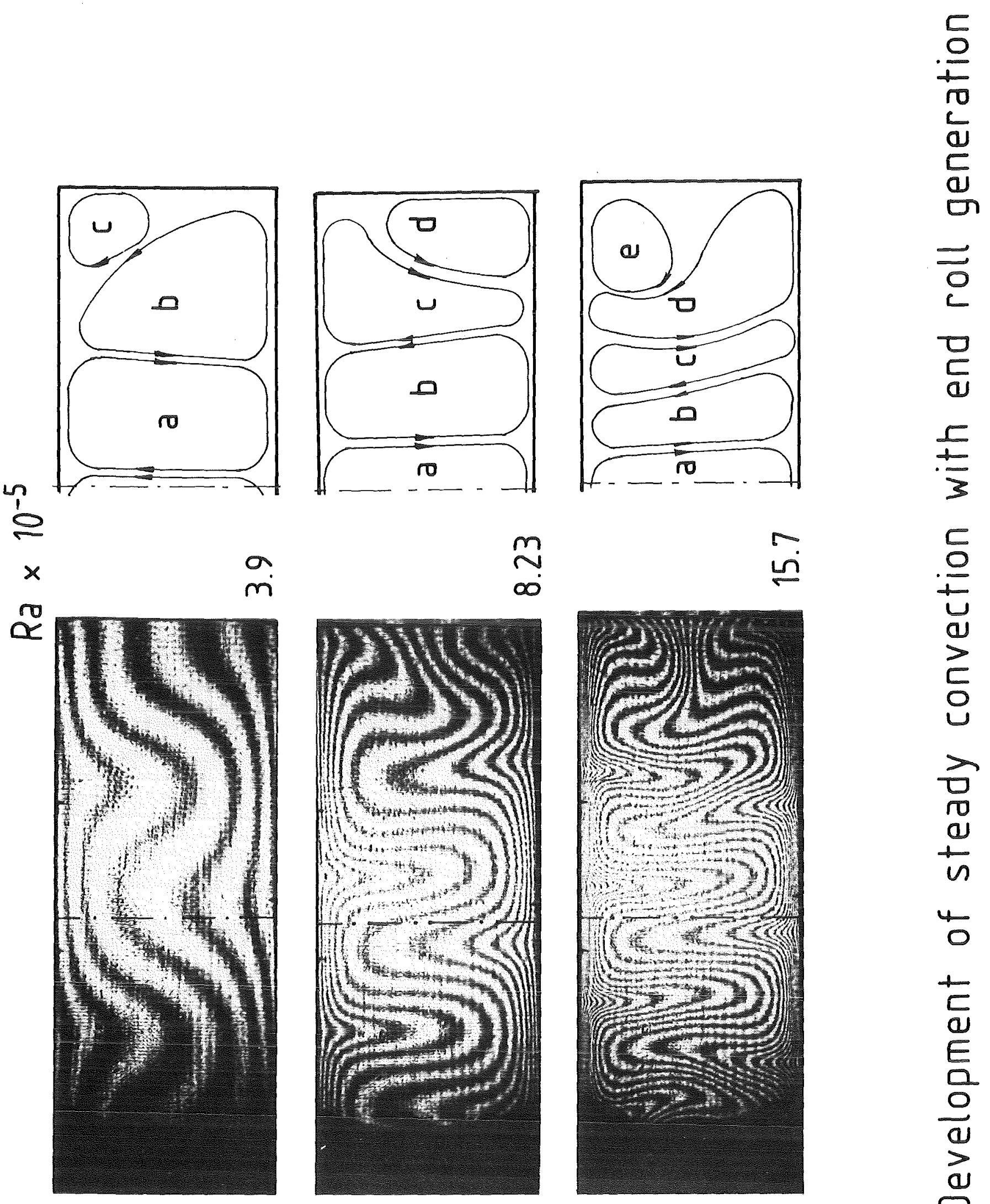

$=$
0
$\square$
$\square$

索

毫
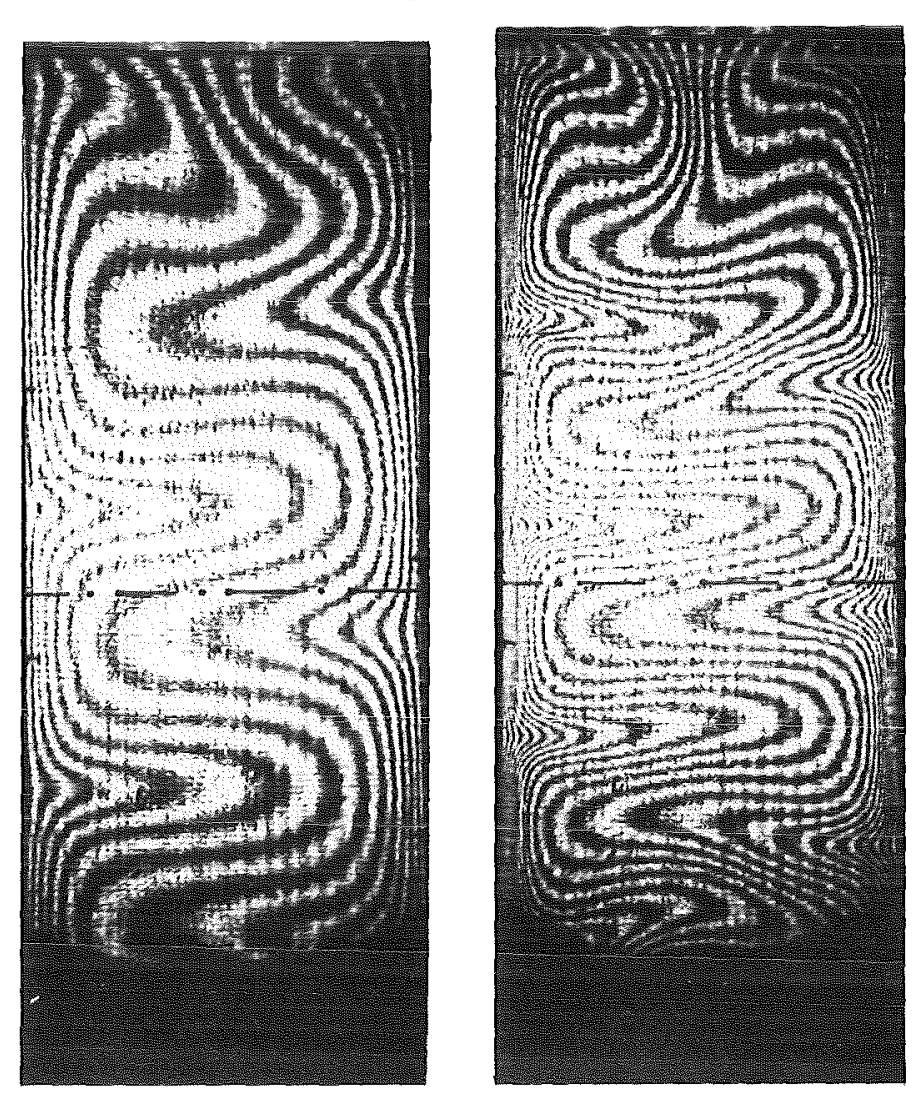

$\stackrel{n}{n}$

0
0
$\frac{0}{n}$

4

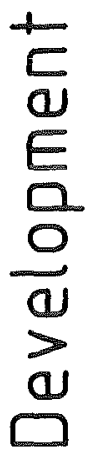

Fig. 2: Development of steady convection with end-roll generation. 
After the onset of convection ( $f$ ig. $2 a$ ) the upflow of the endroll separates from the vertical wall. A recirculation vortex (c) develops in the leading corner at higher Rayleigh-number. This vortex grows with increasing Rayleighnumber to become a fully developed roll cell. Then, the downflow of roll (c) separates from the vertical wall and a new recirculation vortex (d) develops in the lower corner ( $f$ ig. 2b). This vortex grows also to become a fully developed convection cell. Thereafter a third recirculation vortex (e) develops in the upper corner (fig. $2 \mathrm{c}$ ). This vortex, however, grows only until it reaches the midheight of the Hele-Shaw cell, where it builds up a density stratification visualized as area of high fringe density. The corresponding Rayleigh-number represents the final state of steady convection.

From the visualization and especially the stable location of the central upflow (symmetry), it is obvious that three vortices develop almost simultaneously at the other end of the Hele-Shaw cell which is not entirely visualized in the interferograms.

As a remarkable observation during this experiment we note that the number of convection rolls in the Hele-Shaw cell is not constant with increasing Rayleigh number. The flow pattern is controlled by a singular behaviour of the leading corners. From these corners, convection rolls develop and cause a decrease of the wavelength of the rolls already present in the Hele-Shaw ce11. 


\subsection{Uscillatory convection}

The main interest of this study is directed towards the time dependent flow.

The onset of time dependent convection occurred at Ra $\mathrm{Osc}=1.58 \times 10^{6}\left(\mathrm{Ra}^{*}=1\right)$ with a dimensionless period of $\tau=1.02 \times 10^{-2}$. The pattern of the flow oscillations is similar to that observed by Koster \& Müller (1984). A typical temperature signal, exhibiting the characteristics of oszillations just at the onset, is shown in figure $3 a$.

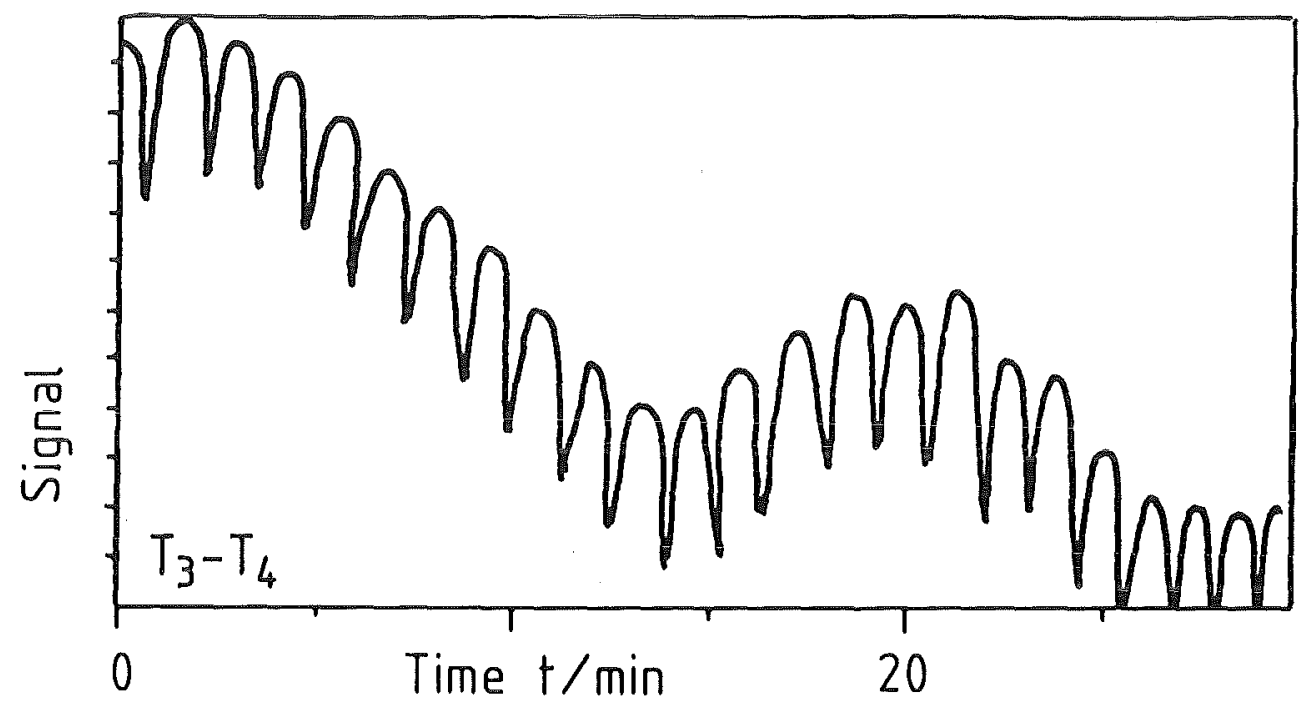

\section{Thermocouple Signal Versus Time}

Fig. 3a: Temperature signal at $\mathrm{Ra}=1.58 \times 10^{6}$

The typical new phenomenon however, in this experiment is that the flow became time dependent only in the regions close to the end walls. Here the vortices have smaller wavenumbers than in the center of the Hele-Shaw cell. The center rolls of high wavenumber remain stable as can be seen from interferograms and from thermocouple signals that monitor the central thermal boundary layers.

Interferograms of one period of oscillation and related qualitative streamline sketches are shown in figure 3. The periodic growth of the corner vortex can be observed in these interferograms. At one time the growing upper vortex is sheared of $f$ by the lower upflow and a cold descending thermal plume forms. The optically measured period of 40 - 50 seconds is confirmed by the frequency analysis of the thermal boundary layer signals. 

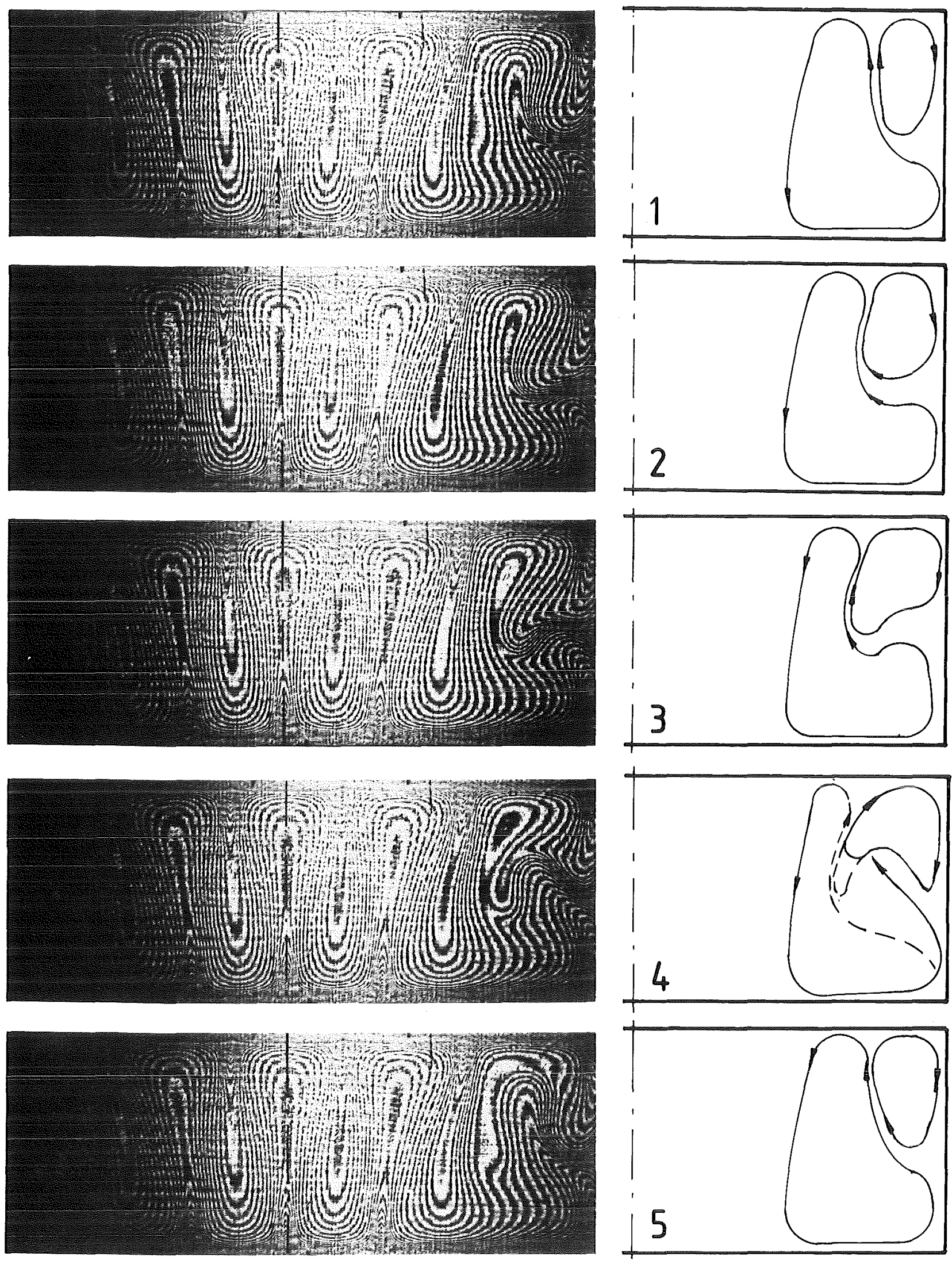

One Period of an end-roll oscillation in a Hele-Shaw cell at Ra $=1.58 \times 10^{6}$ with $\Delta t=47.6 \mathrm{~s}$

Fig. 3: One period of oscillation at $\mathrm{Ra}=1.58 \times 10^{6}$ with period $\Delta t=47.6 \mathrm{~s}$. Interferograms ar taken at $\Delta T=10 \mathrm{~s}$ interval. 
Typical traces of thermocouple signals are shown in figure 4. The signals with reference to the icepoint exhibit more higher frequency noise from the electronics than the differential signals. The autocorrelation coefficients in figure 5 show that the time signals are periodic and have a great coherence. The power density spectra in figure 6, calculated from the autocorrelation coefficients exhibit the typical monoperiodic oscillation pattern as known from relaxation oscillations resulting from thermal boundary layer instabilities. The frequency analysis made only at a few Rayleigh numbers showed that, in the range investigated, all the oscillations at both ends of the Hele-Shaw cell are monoperiodic with one basic frequency and higher harmonics. This monoperiodic behaviour is also expected at Rayleigh numbers between the analyzed measuring points. 


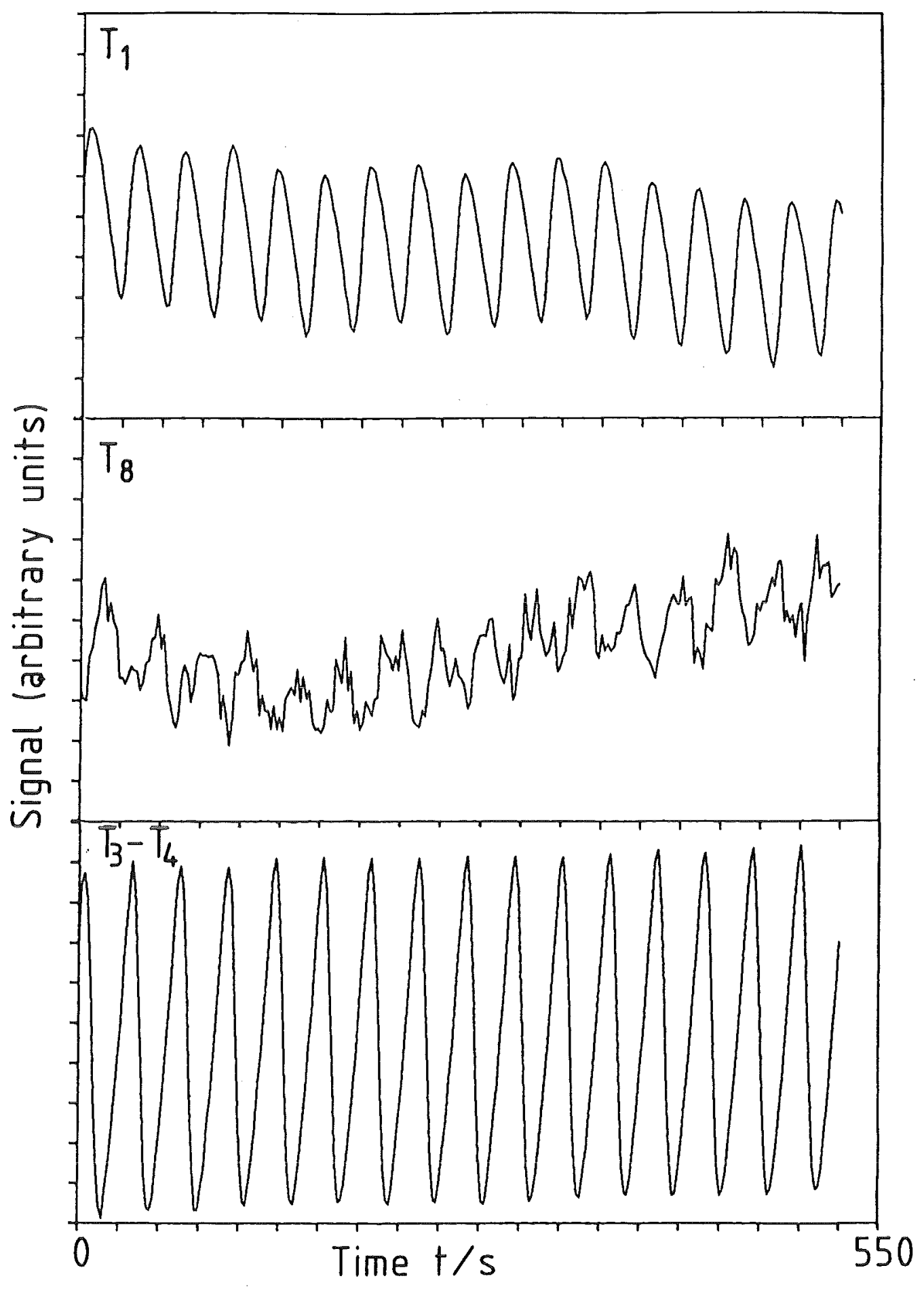

Thermocouple Signals Versus Time

Fig. 4: Temperature signals of $\mathrm{T}_{1}, \mathrm{~T}_{8}$ and $\left(\mathrm{T}_{3}-\mathrm{T}_{4}\right)$ at $\mathrm{Ra}=4.8 \times 10^{6}$ 


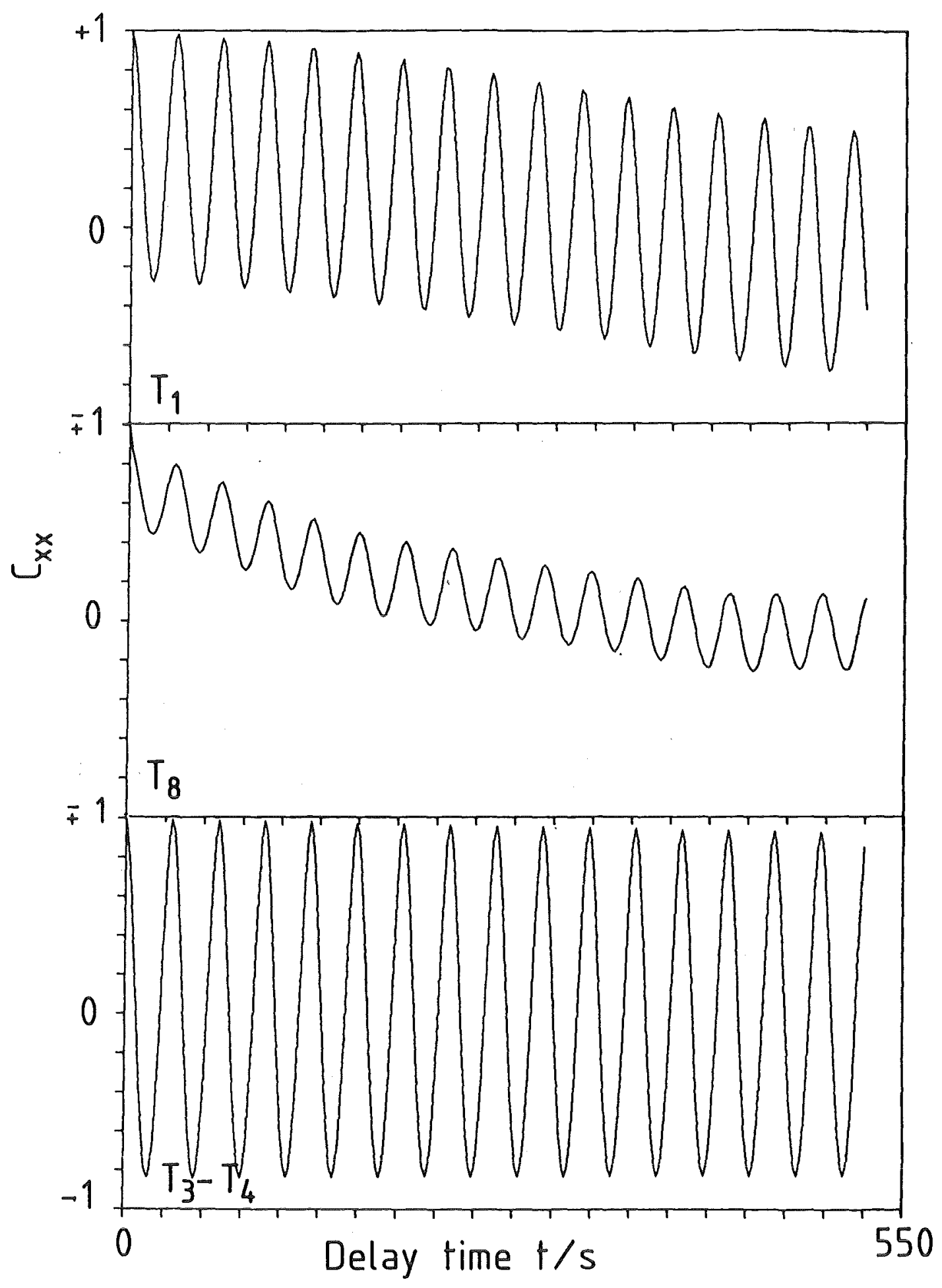

Autocorrelation [oefficients

Fig. 5: Autocorrelation coefficients of the signals in fig. 4. 


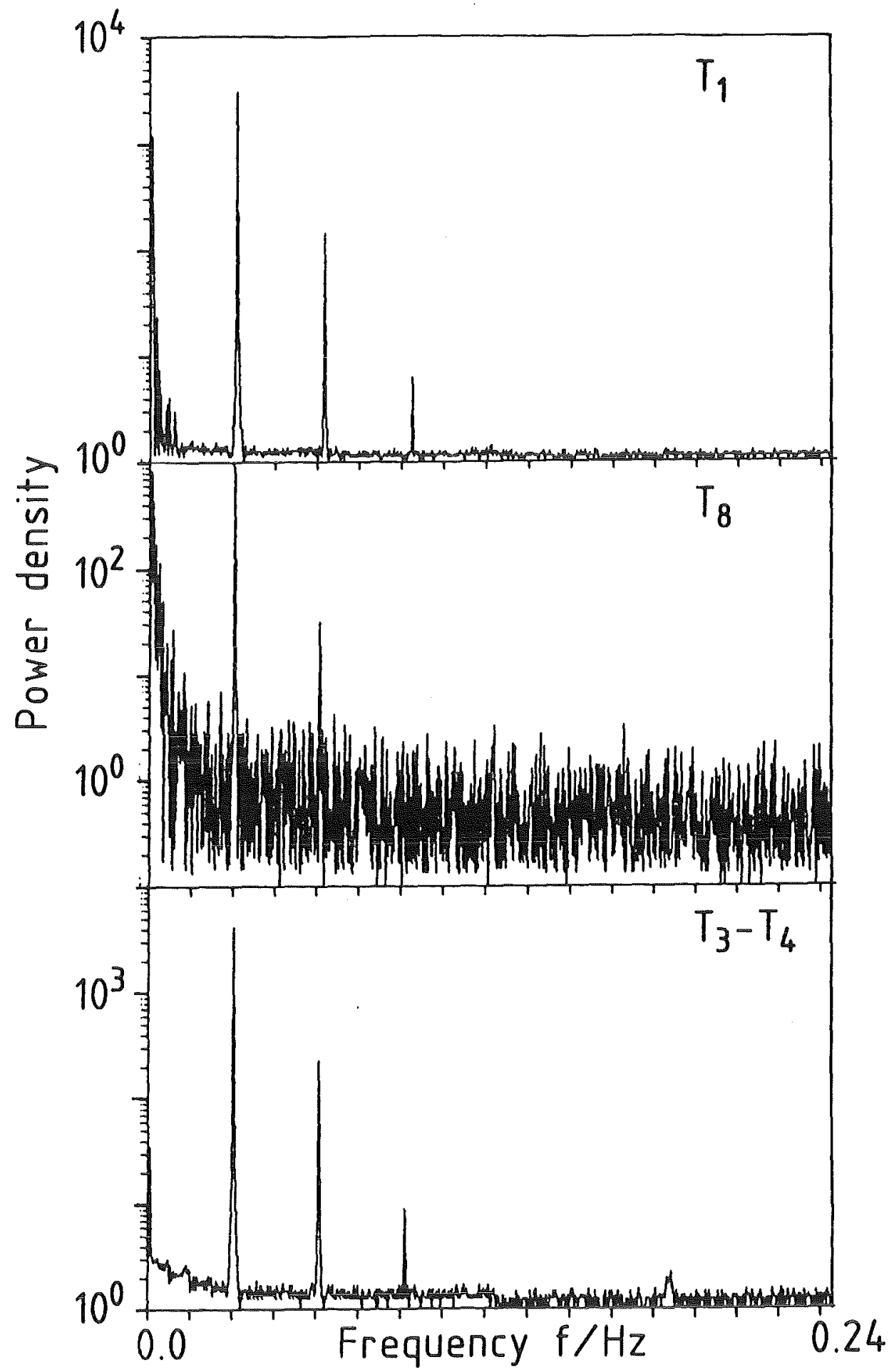

Spectral Density Functions

Fig. 6: Autospectra of the signals in fig. 4. 
The basic frequency of the monoperiodic oscillation, computed as dimensionless period is plotted in figure 7 as a function of the Rayleigh number. Other measured periods taken dircctly from the signal trace are included in the plot. A regression analysis gives the following functional dependence

$$
\tau \sim \mathrm{Ra}^{-1} \cdot 19
$$

this dependence is bounded by the Benard case with $\tau \sim R a^{-2 / 3}$ and by the Hele-Shaw case with $\tau \sim \mathrm{Ra}^{-2}$.

(For detailed derivations of these correlations for instance see Busse \& Whitehead (1974) and Howard (1964)). 


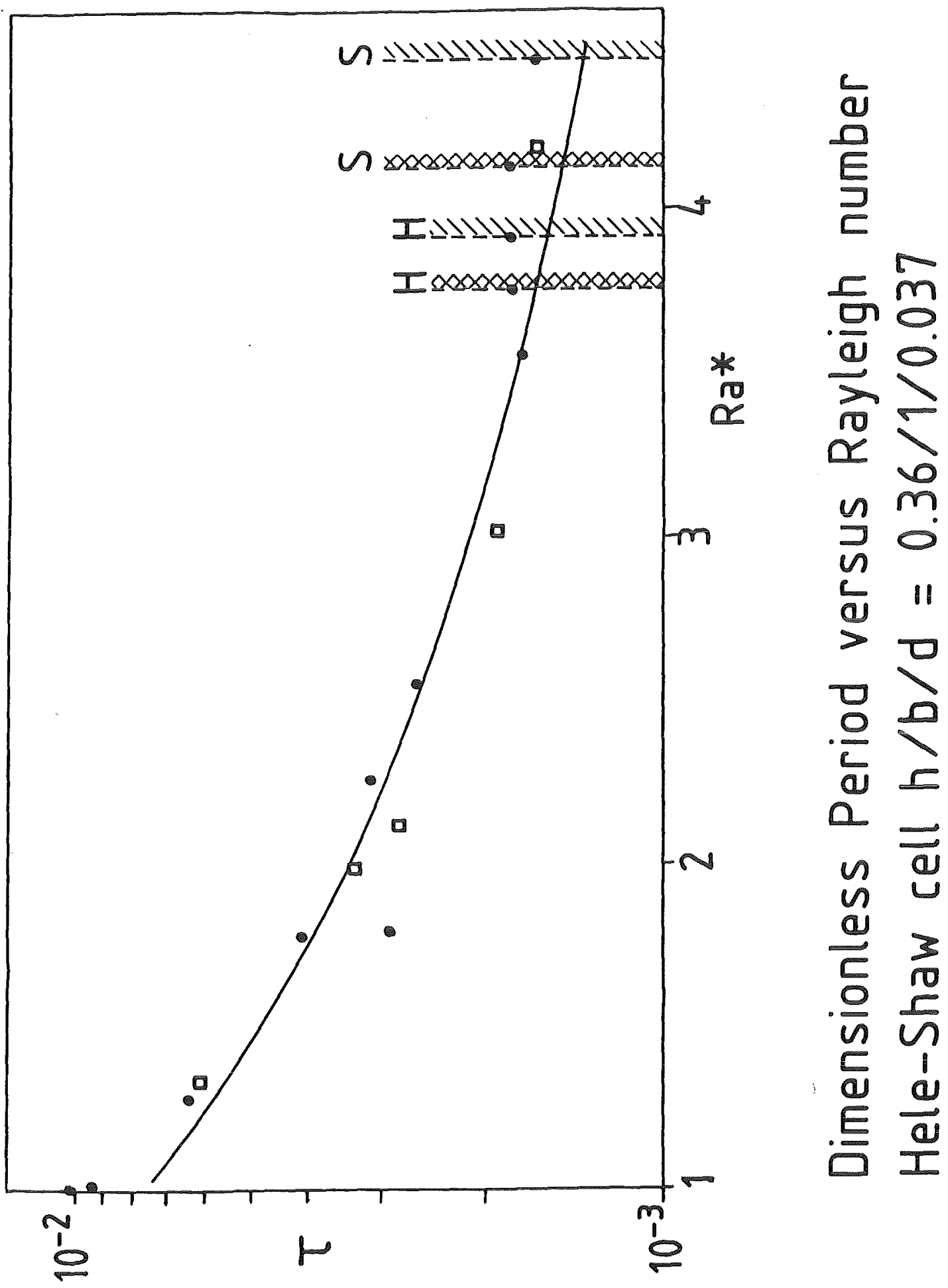

Fig. 7: Dimensionless period $\tau$ as a function of the Rayleigh number $\operatorname{Ra}^{*}=$ $\mathrm{Ra} / \mathrm{Ra} \mathrm{osc}_{\mathrm{S}}$ showing frequency analyzed periods $(\square)$ and data taken from $x t$-recorder ( ). Transition to steady state (S) flow and hysteresis (H) at both sides are also shown. 
The two regions with time-dependent flow are separated by a range of steady flow as shown in figure 3. A cross-correlation (figure 8 ) of the signals measured at one end side of the Hele-Shaw cell, either left or right side, revealed that in the whole range of Rayleigh numbers these signals are well correlated. The plot of the cross correlation function shows also the great coherence of the two signals.

A cross-correlation of any sensor signal at the left end with any sensor signal at the right end of the Hele-Shaw cell revealed that both oscillations, separated by the region of steady convection, are not correlated although the frequencies of oscillation are very close. A typical crosspectrum exhibtting the splitting of the peaks and a plot of the cross-correlation coefficient exhibiting no correlation are shown in figure 9. 

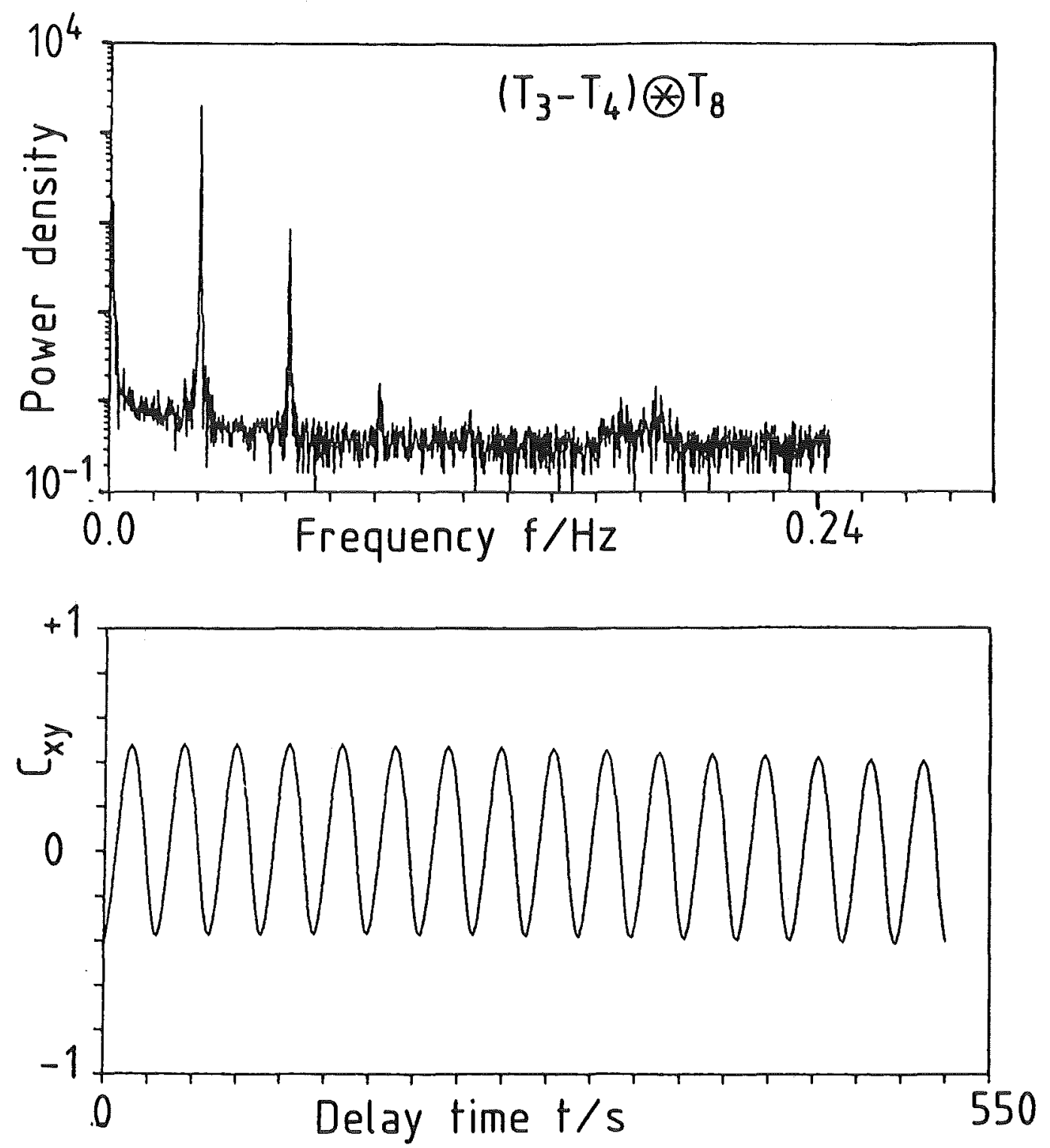

Cross-Spectral Density Function and Cross-Correlation Coefficient. Signals at one end-side.

Fig. 8: Cross-correlation coefficient and cross power density spectrum of the signals $\left(\mathrm{T}_{3}-\mathrm{T}_{4}\right)$ and $\mathrm{T}_{8}$ at one side of the Hele-Shaw cell at $\mathrm{Ra}=$ $4.8 \times 10^{6}$. 

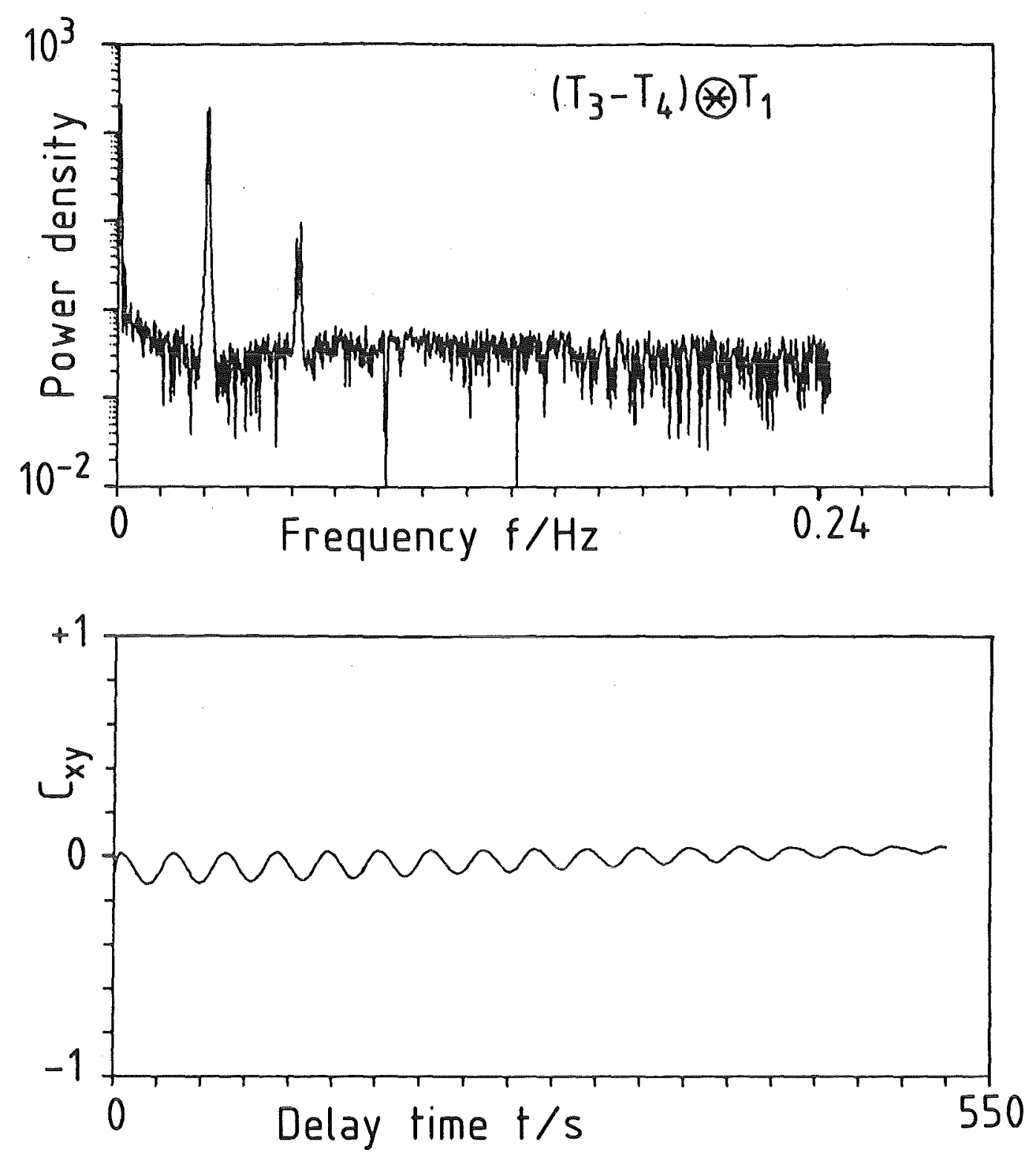

Cross-Spectral Density Function and Cross-Correlation Coefficient. Signals at left and right side.

Fig. 9: Cross-correlation coeffictent and cross power density spectrum of the signals $\left(\mathrm{T}_{3}-\mathrm{T}_{4}\right)$ and $\mathrm{T}_{1}$ which monitor signals at both ends of the Hele-Shaw cell at $\mathrm{Ra}=4.8 \times 10^{6}$. 


\subsection{Reverse transition}

Similar to the experiments by Koster \& Miiller (1984) a reverse transition from time dependent flow to steady flow was observed at high Rayleigh number. It is interesting to note that the oscillations ceased at different Rayleigh numbers at the different sides. On the right side they ceased at $R a=6.54 \times 10^{6}$ and at the left side at $R a=7.08 \times 10^{6}$. Beyond those Rayleighnumbers no time dependent motion was detectable either in the interferogram or in the thermocouple signals. A typical interferogram and the related streamline sketch of the steady state are shown in figure 10. The flow pattern is almost symmetric to the vertical centreline. The end rolls have a lower wavenumber than the center rolls. The shaded area of low fringe density, which means an almost isothermal area, appears only in the case of complete steady flow. At this time there is no physical explanation for this phenomenon as the flow in this area was studied by interferometry only.

When the Rayleigh number is reduced again, time dependent convection begins at the right side at $\mathrm{Ra}=5.95 \times 10^{6}$ and at the left side at $\mathrm{Ra}=6.21 \times 10^{6}$. This is an indication that a pronounced hysteresis effect is related to this reverse transition.
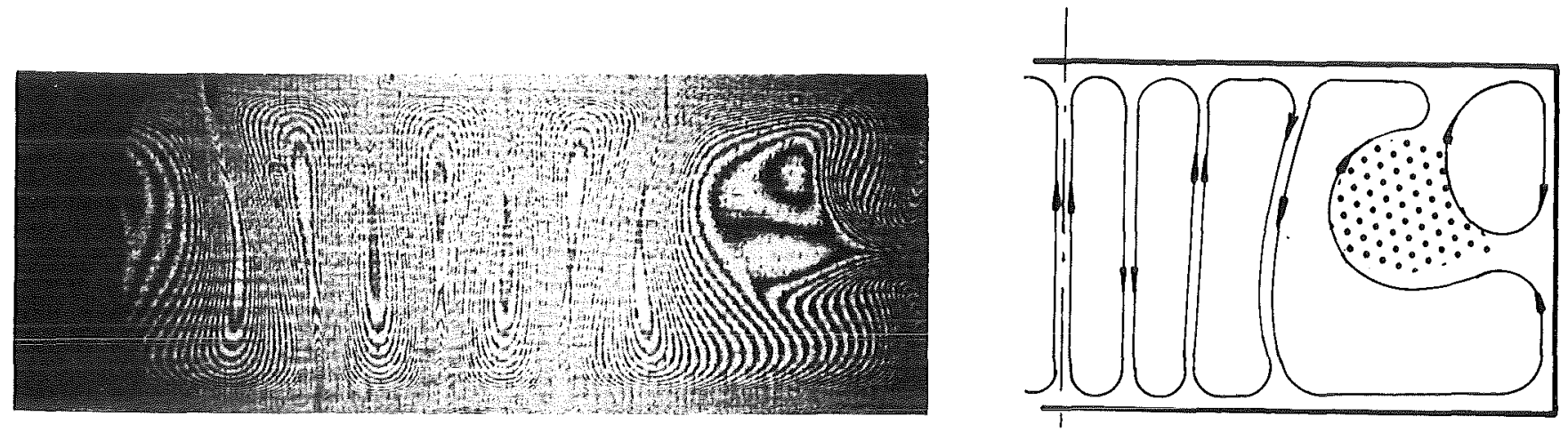

\section{Steady convection at $\mathrm{Ra}>6.56 \times 10^{6}$}

Fig. 10: Steady convection flow at $\mathrm{Ra}>6.56 \times 10^{6}$. 


\section{Discussion}

The onset of convection in the Hele-Shaw cell is conform to the theoretical bounds given by Frick \& Clever (1980). A new finding is the generation of vortices in the leading end corners of the cavity. This is a similar observation as reported by Elder (1967) in a llele-Shaw cell with other thermal boundary conditions. In other Hele-Shaw cells with the same thermal boundary conditions but with lower Prandt 1 number fluid $(\operatorname{Pr}=7)$ and a smaller depth $(d=1 \mathrm{~mm})$ the $i n c r e a s e$ in wavenumber with increasing values of $R a$ was not observed (Koster \& Müller 1982).

After a regime with time-dependent convection that we discuss below, a reverse transition to a steady flow, exhibiting a hysteresis, was observed at high Ra. This finding is similar to the observations made by Koster \& Muiller (1984)。

The new finding that regions of steady flow can coexist with regions of timedependent flow may be explained by the theoretical results of Frick \& Muller (1983). They investigate the stability of convective flow as a function of the wavenumber of the rolls. According to their stability diameter the central region is expected to be steady while the end rolls exhibit oscillations as the wavenumber of the central rolls is much higher than that of the end rolls.

It is obvious from these experiments that the end walls have a great influence on the stability of the flow. But it was not possible to find out whether the oscillations start at the density interface at midheight of the cell or in the horizontal thermal boundary layers. An instability in one of these regions could induce the instability into the other and perform a frequency locked and highly correlated oscillation as analyzed. We found that at the onset of oscillations, relaxation oscillations exist which are interpreted as thermal boundary layer instabilities which develop at the horizontal boundaries. The Howard model (1964) of the development of time dependent convection at high Rayleigh number is thus accepted as driving mechanism of the oscillations. 
Koster \& Müller (1982) observed that in a Hele-Shaw cell of large horizontal extent containing many convection rolls, the positions of the free vertical boundaries of the convections fluctuate, i.e. the roll cells expand temporarily at the expense of a neighbouring cell or give space to it. These fluctuations imply a recurrent perturbation and the flow oscillation becomes nonperiodic. In the present experiment there is no perturbation of the oscillating roll cell by the steady neighbouring roll cells, which is considered the cause for the oscillations to remain monoperiodic relaxation oscillations at any Rayleigh number. This physical arguing is backed by the correlation analysis showing that the oscillations in the two end regions are not correlated.

Considering results by Dubois \& Berge (1981) as well as those by Koster \& Muller (1984) and the present investigation, it is apparent that different thermal boundary layer instabilities develop individual frequencies and interact to produce flow oscillations that may be more complicated than the monoperiodic oscillations. This might explain why the routes to turbulence in small convection boxes with two convection cells, often develop in a Ruelle \& Takens (1971) sequence like monoperiodic, quasiperiodic (biperiodic, frequency locked), turbulent. In extended fluid layers with more than two roll cells of somewhat different wavenumbers many different oscillators at each horizontal boundary provide, with mutual interaction (see Koster \& Müller 1982), the instantaneous transition from steady to turbulent flow. Frick \& Müller (1983) have shown on the other hand in numerical experiments that in case many roll cells of constant and equal wavenumber interact the oscillations are always periodic. These observations indicate that time dependent convection in high Prandt 1 number flow is governed by individual thermal boundary layer instabilities developing at individual frequencies. Nonlinear interaction of these instabilities in the fluid layer provides the different oscillations and transitions between them.

The model suggested here, however, does in general not exhibit period doubling (Feigenbaum 1979) and intermittent (Manneville \& Pomeau 1979) routes to turbulence. 


\section{Summary}

Convection experiments were performed in a low conductivity Hele-Shaw cell with the aspect ratio $h / b / d=0.36 / 1 / 0.037$. The fluid of Prandt number Pr $\approx$ 235 was heated from below and cooled from above. A total of 8 thermocouples monitored thermal perturbations in the horizontal thermal boundary layers.

It is observed that three regions may coexist at one Rayleigh number in a fluid layer. One central region is steady and the two end regions are time dependent. The two individual oscillations are monoperiodic and never correlated at any Rayleigh number of the time dependent regime.

We suggest that in our case (high Prandt number fluid in gaps) the route to turbulence may be controlled by precise selection of interacting unstable thermal boundary layers. 


\section{References}

Ahlers, G. and Behringer, R.P., Phys. Rev. Lett. 40 (1978) 712.

Berge, P. and Dubois, M., Optics Commun. 19(1976)129.

Busse, F.H. and Whitehead, J.A., J. Fluid Mech.66(1974)67.

Busse, F.H., Hydrodynamic Instaoilities and the Transition to Turbulence, H.L. Swinney \& J.P. Gollub, eds., (Springer) 1981, p. 97.

Dubois, Mo and Berge, $P_{i}$, Phys. Lett. 76A (1980) 53.

Dubois, M. and Berge, P., J. Physique 42(1981)167.

Elder, J.W., J. Fluid. Mech. 27 (1967)29.

Feigenbaum, M.J., Phys.Lett. i4A (1979)375.

Frick, H. and Clever, R.M., ZAMP 31 (1980)502.

Frick, H. and Müller, U., J. Fluid. Mech. 126(1983)521.

Gollub, J.P. and Benson, S.V., J. Fluid Mech. 100 (1980)449.

Howard, L॰N., Proce 11 th Int, Congr。 Appl. Mech. H. Görtler, ed॰, (Springer) 1966, p. 1109

Koschmieder, E.L., Order and Fluctuations in Equilibrium and Non-Equilibrium Statistical Mechanics, E. Nicolis, G. Dewel \& J.W. Turner, eds., (Wiley) 1981, p. 159.

Koster, J.N. and Müller, U., J. Fluid Mech. 125(1982)429.

Koster, J.N. and Müller, U., J. Fluid Mech. 139(1984)363.

Krishnamurti, R., J. Fluid Mech. 42(1970)309。 
Libchaber, A. and Maurer, J., J. Physique Collog. 4l (1980)C3-51.

Manneville, P. and Pomeau, Y., Phys. Lett. 75A (1979),1.

Ruelle, D. and Takens, F., Commun. Math. Phys. 20(1971)167. 